\title{
Symmetry preserving partial pole assignment for the standard and the generalized eigenvalue problems
}

\author{
S. Elhay ${ }^{1}$
}

(Received 29 August 2006; revised 12 July 2007)

\begin{abstract}
Many real-life state feedback control systems are modelled by a set of single-input, time-invariant linear equations. Often, these lead to a pair of matrices which are real and symmetric. The pole assignment problem requires us to find a state feedback control which gives the closed loop matrices a prescribed set of poles. Commonly used controls produce a closed loop matrix which is no longer symmetric. This article presents an explicit solution for a new symmetry preserving partial pole assignment method for the generalised eigenvalue problem and for the standard eigenvalue problem. The methods are demonstrated on illustrative examples and can produce computationally accurate solutions for even quite large systems.
\end{abstract}

See http://anziamj.austms.org.au/ojs/index.php/ANZIAMJ/article/view/106 for this article, (c) Austral. Mathematical Soc. 2007. Published July 22, 2007. ISSN 14468735 


\section{Contents}

1 Introduction

C265

2 Symmetry preserving pole assignment for a symmetric matrix

C269

3 Symmetry preserving pole assignment for a symmetric pair of matrices

$\mathrm{C} 273$

4 Conclusions

$\mathrm{C} 277$

References

$\mathrm{C} 277$

\section{Introduction}

Consider a system modelled by the single-input time-invariant linear equation

$$
\boldsymbol{B} \dot{\boldsymbol{x}}(t)=\boldsymbol{A} \boldsymbol{x}(t)+\boldsymbol{b} u(t), \quad \boldsymbol{x}\left(t_{0}\right)=\boldsymbol{x}_{0},
$$

where $\boldsymbol{x}(t) \in \mathbb{R}^{n}$ represents the system's state, $\boldsymbol{x}_{0} \in \mathbb{R}^{n}$ the state vector at the initial time $t=t_{0}, u(t)$ is an input control for the system, and $\boldsymbol{b} \in \mathbb{R}^{n}$ and the matrices $\boldsymbol{A}, \boldsymbol{B} \in \mathbb{R}^{n \times n}$ are constant. When $\boldsymbol{b}=\mathbf{0}$ Equation (1) is referred to as the open-loop system. In the classical pole placement or pole assignment problem we are required to find a state feedback control $u(t)=\boldsymbol{f}^{T} \boldsymbol{x}(t)-\boldsymbol{g}^{T} \dot{\boldsymbol{x}}(t)$ such that the closed loop system

$$
\left(\boldsymbol{B}+\boldsymbol{b} \boldsymbol{g}^{T}\right) \dot{\boldsymbol{x}}(t)=\left(\boldsymbol{A}+\boldsymbol{b} \boldsymbol{f}^{T}\right) \boldsymbol{x}(t)
$$

has prescribed poles. In the language of linear algebra we are required to find vectors $\boldsymbol{f}, \boldsymbol{g}$ such that the matrix pencil $\left(\boldsymbol{A}+\boldsymbol{b} \boldsymbol{f}^{T}\right)-\lambda\left(\boldsymbol{B}+\boldsymbol{b} \boldsymbol{g}^{T}\right)$ has the prescribed spectrum. An important special case of the pole placement 
problem is that of stabilisation in which all the poles of the closed loop system are required be in the open left half-plane.

Many real life systems which are modelled by finite elements lead to a pair of matrices $\boldsymbol{A}, \boldsymbol{B}$ which, in addition to being real, are symmetric. For these systems the process of applying a control of the type in (2) produces a closed loop matrix which is no longer symmetric. However, it is sometimes necessary (the control of vibratory systems by passive elements is an example) for the closed-loop system to satisfy a reciprocity law: the force at $x_{1}$ due to a unit displacement at $x_{2}$ should equal the force at $x_{2}$ due to a unit displacement at $x_{1}$. For systems such as these the available controls are more restricted than those for (2) and one such restriction is that they may need to be symmetric. The symmetric closed loop pencil of the form

$$
P_{c}(\lambda)=\left(\boldsymbol{A}+\alpha \boldsymbol{u} \boldsymbol{u}^{T}\right)-\lambda\left(\boldsymbol{B}+\beta \boldsymbol{u} \boldsymbol{u}^{T}\right),
$$

$\alpha, \beta$ scalars, has been considered before and Ram [7] solved the pole placement problem modelling a continuous version of the problems addressed here. Datta [1] reviewed some recent developments in computational methods for several inverse eigenvalue problems for matrix quadratic pencils.

Suppose $\boldsymbol{A}, \boldsymbol{B} \in \mathbb{R}^{n \times n}$ are both symmetric and at least one of them is positive definite. Such a pair is said to be symmetric definite. In this article we consider pole placement problems for systems of two types:

1. the standard symmetric pole placement problem (equivalent to $\boldsymbol{B}=\boldsymbol{I}$, $\alpha= \pm 1$ and $\beta=0$ in (3)) for the system $\boldsymbol{A}+\sigma \boldsymbol{v} \boldsymbol{v}^{T}, \sigma= \pm 1$; and

2. the generalized symmetric definite pair pole placement problem for the system $\boldsymbol{A}+\alpha \boldsymbol{u} \boldsymbol{u}^{T}-\lambda\left(\boldsymbol{B}+\beta \boldsymbol{u} \boldsymbol{u}^{T}\right), \quad \alpha, \beta$ scalars.

It is well known that the eigenvalues and eigenvectors of a real symmetric matrix $\boldsymbol{A}$ and those of a real symmetric definite pair $\boldsymbol{A}, \boldsymbol{B}$ are real. Furthermore, the eigenvalues of $\boldsymbol{A}$ and those of $\boldsymbol{A}+\sigma \boldsymbol{v} \boldsymbol{v}^{T}$ satisfy the following interlacing property $[3, \S 8.6 .3$, e.g.]: 
Let $\boldsymbol{A} \in \mathbb{R}^{n \times n}$ be symmetric and let the spectrum of the pencil $P_{o}(\lambda)=$ $\boldsymbol{A}-\lambda \boldsymbol{I}$, denoted by $\sigma\left(P_{o}\right)=\left\{\lambda_{j}\right\}_{j=1}^{n}$, be such that $\lambda_{1} \leq \lambda_{2} \leq \cdots \leq$ $\lambda_{n}$. Define, for simplicity, $\lambda_{n+1}=\mu_{n+1}=\infty$ and let $\sigma= \pm 1$. Then the eigenvalues $\left\{\mu_{j}\right\}_{j=1}^{n}$ of $\boldsymbol{A}+\sigma \boldsymbol{v} \boldsymbol{v}^{T}$ satisfy

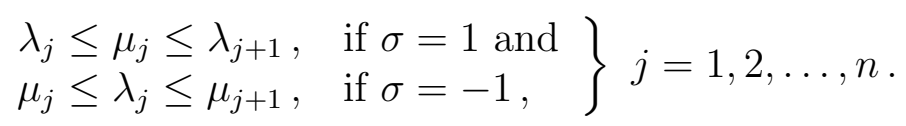

Similarly, the spectrum of the pencil $P_{o}(\lambda)=\boldsymbol{A}-\lambda \boldsymbol{B}[2]$, denoted by

$$
\sigma(\boldsymbol{A}, \boldsymbol{B})=\left\{\lambda_{j}\right\}_{j=1}^{n}
$$

and assumed here to be ordered $\lambda_{1} \leq \lambda_{2} \leq \cdots \leq \lambda_{n}$, and the spectrum $\left\{\mu_{j}\right\}_{j=1}^{n}$ of the modified generalized symmetric pair satisfy the interlacing property:

$$
\left.\begin{array}{cll}
\alpha / \beta \leq \mu_{1} \leq \lambda_{1} & \text { if } & \alpha / \beta \leq \lambda_{1} \\
\lambda_{j} \leq \mu_{j} \leq \lambda_{j+1} & \text { if } \quad \lambda_{j} \leq \alpha / \beta \\
\lambda_{j} \leq \mu_{j} \leq \alpha / \beta \leq \mu_{j+1} \leq \lambda_{j+1} & \text { if } \quad \lambda_{j} \leq \alpha / \beta \leq \lambda_{j+1} \\
\lambda_{j} \leq \mu_{j+1} \leq \lambda_{j+1} & \text { if } \quad \alpha / \beta \leq \lambda_{j} \\
\lambda_{n} \leq \mu_{n} \leq \alpha / \beta & \text { if } \quad \lambda_{n} \leq \alpha / \beta
\end{array}\right\} j=1,2, \ldots, n
$$

Clearly then, only poles which satisfy the appropriate interlacing property can be assigned by these kinds of symmetric rank-one updates.

This article presents a new explicit formula for the vector $\boldsymbol{u}$ which assigns either the whole spectrum, or part of the spectrum, of the closed loop generalized symmetric pair system. This is done by computing symmetry preserving rank-one updates which assign the spectrum of the generalized eigenvalue system. Where only a part of the spectrum is assigned the eigenvalues not replaced remain unchanged and there is no possibility of spillover, the phenomenon in which eigenvalues which it is intended to preserve are disturbed by the assignment and move to the right half of the plane. More importantly, it is not necessary to know the whole spectrum of the openloop system: only those $\lambda_{j}$ which are to be replaced need to be known. The 
fact that the $\mu_{j}$ need to satisfy (6) will not usually present a difficulty even though not all $\lambda_{j}$ are known. On the way to the new formula we briefly review Lowner's method [6] which solves Problem 1 below and extend its application to symmetry preserving partial pole assignment for the standard eigenvalue problem (so far apparently not noted for pole placement and model updating). More precisely, we extend (to the case when $r<n$ ) Lowner's method to solve

Problem 1 Given (a) $\boldsymbol{A} \in \mathbb{R}^{n \times n}$, symmetric with spectrum $\lambda_{1} \leq \lambda_{2} \leq$ $\cdots \leq \lambda_{n}$; (b) a scalar $\sigma= \pm 1$; (c) a set of real numbers $\left\{\mu_{j}\right\}_{j=1}^{r}, r \leq n$ which satisfy (4); find a vector $\boldsymbol{v} \in \mathbb{R}^{n}$ which is such that $\boldsymbol{A}+\boldsymbol{v} \boldsymbol{v}^{T}$ has spectrum $\sigma\left(\boldsymbol{A}+\boldsymbol{v} \boldsymbol{v}^{T}\right)=\left\{\mu_{1}, \mu_{2}, \ldots, \mu_{r}, \lambda_{r+1}, \lambda_{r+2}, \ldots, \lambda_{n}\right\}$,

and we present an explicit solution to

Problem 2 Given (a) matrices $\boldsymbol{A}, \boldsymbol{B} \in \mathbb{R}^{n \times n}$, both symmetric and $\boldsymbol{B}$ positive definite, such that the spectrum of $P(\lambda)=\boldsymbol{A}-\lambda \boldsymbol{B}$ satisfies $\lambda_{1} \leq$ $\lambda_{2} \leq \lambda_{3} \ldots \leq \lambda_{n}$; (b) a pair of scalars $\alpha \geq 0, \beta>0$ such that $\alpha / \beta \neq \lambda_{j}$ any $j$; (c) a set of real scalars $\left\{\mu_{j}\right\}_{j=1}^{r}, r \leq n$ which satisfy (6); find a vector $\boldsymbol{u} \in \mathbb{R}^{n}$ such that the spectrum of the pencil $P_{c}(\lambda)=\boldsymbol{A}+\alpha \boldsymbol{u} \boldsymbol{u}^{T}-\lambda\left(\boldsymbol{B}+\beta \boldsymbol{u} \boldsymbol{u}^{T}\right)$ is $\sigma\left(\boldsymbol{A}+\alpha \boldsymbol{u} \boldsymbol{u}^{T}, \boldsymbol{B}+\beta \boldsymbol{u} \boldsymbol{u}^{T}\right)=\left\{\mu_{1}, \mu_{2}, \ldots, \mu_{r}, \lambda_{r+1}, \lambda_{r+2}, \ldots, \lambda_{n}\right\}$.

Preliminary numerical experiments in which the methods have been applied to a range of matrix pairs of dimension up to 1024, even with spectra to be assigned that are very close to the existing spectra (a numerically challenging problem), indicate that the method is remarkably accurate. More detailed numerical testing will be the subject of a later article.

In Section 2 we introduce the method, derive the explicit solution and illustrate its application on a full pole assignment example and a partial pole assignment problem. In Section 3 we derive the explicit solution for the generalized definite symmetric pair problem and illustrate its application to 
full and partial pole assignment for a definite symmetric pair. We conclude with some remarks on further work.

\section{Symmetry preserving pole assignment for a symmetric matrix}

Here we briefly review the secular equation for a symmetric matrix updated by a rank-one matrix.

Let $\mu, \boldsymbol{y}$ be an eigenpair of the closed loop system matrix $\left(\boldsymbol{A}+\sigma \boldsymbol{v} \boldsymbol{v}^{T}\right) \boldsymbol{y}=$ $\mu \boldsymbol{y}$, and suppose that we have the eigendecomposition $\boldsymbol{A}=\boldsymbol{Q} \boldsymbol{\Lambda} \boldsymbol{Q}^{T}, \boldsymbol{\Lambda}=$ $\operatorname{diag}\left\{\lambda_{1}, \lambda_{2}, \ldots, \lambda_{n}\right\}$ and $\boldsymbol{Q}$ orthogonal. Thus, $\left(\boldsymbol{\Lambda}+\sigma \boldsymbol{Q}^{T} \boldsymbol{v} \boldsymbol{v}^{T} \boldsymbol{Q}\right) \boldsymbol{Q}^{T} \boldsymbol{y}=\mu \boldsymbol{Q}^{T} \boldsymbol{y}$ or, denoting $\hat{\boldsymbol{v}}=\boldsymbol{Q}^{T} \boldsymbol{v}$ and $\hat{\boldsymbol{y}}=\boldsymbol{Q}^{T} \boldsymbol{y}$, we get $\left(\boldsymbol{\Lambda}+\sigma \hat{\boldsymbol{v}} \hat{\boldsymbol{v}}^{T}\right) \hat{\boldsymbol{y}}=\mu \hat{\boldsymbol{y}}$. Rearranging gives

$$
\sigma \hat{\boldsymbol{v}} \hat{\boldsymbol{v}}^{T} \hat{\boldsymbol{y}}=(\mu \boldsymbol{I}-\boldsymbol{\Lambda}) \hat{\boldsymbol{y}} .
$$

Denote by $\boldsymbol{e}_{j}$ the $j$ th column of an identity of appropriate dimension. Provided that $\boldsymbol{e}_{j}^{T} \boldsymbol{v} \neq 0$ for any $j=1,2, \ldots, n$, then three things follow [3, §8.6.3,e.g.]: (a) $(\mu \boldsymbol{I}-\boldsymbol{\Lambda})$ is invertible; (b) $\hat{\boldsymbol{v}}^{T} \hat{\boldsymbol{y}} \neq 0$; (c) The eigenvector $\hat{\boldsymbol{y}}$ of the rank-one modified system is a scalar multiple of $(\mu \boldsymbol{I}-\boldsymbol{\Lambda})^{-1} \hat{\boldsymbol{v}}$.

Denoting $p=\hat{\boldsymbol{v}}^{T} \hat{\boldsymbol{y}}$ in (7) we get $\sigma(\mu \boldsymbol{I}-\boldsymbol{\Lambda})^{-1} \hat{\boldsymbol{v}} p=\hat{\boldsymbol{y}}$ and multiplying both sides by $\hat{\boldsymbol{v}}^{T}$ gives the secular equation

$$
\sigma \hat{\boldsymbol{v}}^{T}(\mu \boldsymbol{I}-\boldsymbol{\Lambda})^{-1} \hat{\boldsymbol{v}}=1,
$$

sometimes written as

$$
\sigma \sum_{k=1}^{n} \frac{\hat{v}_{k}^{2}}{\mu-\lambda_{k}}=1 .
$$

This equation is usually used to find the eigenvalues of the modified system from the known eigenvalues $\left\{\lambda_{j}\right\}$ and the rank-one correction vector $\hat{\boldsymbol{v}}$. Our 
interest here, however, is in the inverse problem and we now show how to use the secular equation to determine the vector $\hat{\boldsymbol{v}}$.

We note here, for later use, that if $\boldsymbol{e}_{j}^{T} \hat{\boldsymbol{v}}=0$ for some $j$, then $\left(\lambda_{j}, \boldsymbol{e}_{j}\right)$ is an eigenpair of the modified system because then $\left(\left(\boldsymbol{\Lambda}+\sigma \hat{\boldsymbol{v}} \hat{\boldsymbol{v}}^{T}\right)-\lambda_{j} \boldsymbol{I}\right) \boldsymbol{e}_{j}=$ $\Lambda \boldsymbol{e}_{j}-\lambda_{j} \boldsymbol{e}_{j}=0$. In such a case we can deflate the problem to one with dimension $n-1$. Importantly, the secular equation then has $n-1$ terms and $n-1$ solutions and so the modification has left the eigenvalue $\lambda_{j}$ of the original system unchanged.

We now briefly review Lowner's method [4] and then show how to apply it in the case of partial pole assignment.

For simplicity let us consider first the full pole placement problem in which we want to assign all the poles of the closed loop system. Thus we are given $n$ real numbers $\left\{\mu_{j}\right\}_{j=1}^{n}$ which interlace the eigenvalues of $\boldsymbol{A}$ in the sense of (4) and we want to construct a vector $\hat{\boldsymbol{v}}$ which achieves this. Substituting $\mu=\mu_{j}$, for each $j=1,2, \ldots, n$ into (9) gives a set of $n$ equations which are linear in the unknowns $\hat{v}_{j}^{2}$. The matrix for this system of equations

$$
\left[\begin{array}{cccc}
\frac{1}{\mu_{1}-\lambda_{1}} & \frac{1}{\mu_{1}-\lambda_{2}} & \cdots & \frac{1}{\mu_{1}-\lambda_{n}} \\
\overline{\mu_{2}-\lambda_{1}} & \frac{1}{\mu_{2}-\lambda_{2}} & \cdots & \frac{1}{\mu_{2}-\lambda_{n}} \\
\vdots & & & \vdots \\
\frac{1}{\mu_{n}-\lambda_{1}} & \frac{1}{\mu_{n}-\lambda_{2}} & \cdots & \frac{1}{\mu_{n}-\lambda_{n}}
\end{array}\right]\left[\begin{array}{c}
\hat{v}_{1}^{2} \\
\hat{v}_{2}^{2} \\
\vdots \\
\hat{v}_{n}^{2}
\end{array}\right]=\left[\begin{array}{c}
1 \\
1 \\
\vdots \\
1
\end{array}\right],
$$

called a Cauchy matrix, $\boldsymbol{C}=\left[c_{i j}\right]=\frac{1}{\mu_{i}-\lambda_{j}}$, has inverse

$$
\boldsymbol{C}^{-1}=\left[b_{i j}\right]=\frac{\prod_{k=1}^{n}\left(\lambda_{i}-\mu_{k}\right) \prod_{k=1}^{n}\left(\mu_{j}-\lambda_{k}\right)}{\left(\lambda_{i}-\mu_{j}\right) \prod_{k=1, k \neq i}^{n}\left(\lambda_{i}-\lambda_{k}\right) \prod_{k=1, k \neq j}^{n}\left(\mu_{j}-\mu_{k}\right)} .
$$

Thus we can write, for $i=1,2, \ldots, n$,

$$
\hat{v}_{i}^{2}=-\frac{\prod_{k=1}^{n}\left(\lambda_{i}-\mu_{k}\right)}{\prod_{k=1, k \neq i}^{n}\left(\lambda_{i}-\lambda_{k}\right)} \sum_{j=1}^{n} \frac{\prod_{k=1, k \neq i}^{n}\left(\mu_{j}-\lambda_{k}\right)}{\prod_{k=1, k \neq j}^{n}\left(\mu_{j}-\mu_{k}\right)} .
$$


Using the identity $[5, \S 1.2 .3,33]$

$$
\sum_{j=1}^{n} \frac{\mu_{j}^{r}}{\prod_{k=1, k \neq j}^{n}\left(\mu_{j}-\mu_{k}\right)}= \begin{cases}0, & \text { if } 0 \leq r<n-1, \\ 1, & \text { if } r=n-1,\end{cases}
$$

we immediately see that

$$
\sum_{j=1}^{n} \frac{\prod_{k=1, k \neq i}^{n}\left(\mu_{j}-\lambda_{k}\right)}{\prod_{k=1, k \neq j}^{n}\left(\mu_{j}-\mu_{k}\right)}=1, \quad i=1,2, \ldots, n,
$$

from which it quickly follows that

$$
\hat{v}_{i}^{2}=-\frac{\prod_{k=1}^{n}\left(\lambda_{i}-\mu_{k}\right)}{\prod_{k=1, k \neq i}^{n}\left(\lambda_{i}-\lambda_{k}\right)}, \quad i=1,2, \ldots, n .
$$

Thus there are $2^{n}$ different solutions in view of the two sign choices that can be made for each component of the solution vector $\hat{\boldsymbol{v}}$.

If we consider only $\times, \div$ operations then using (12) costs $2 n(n-1)$ operations and so is computationally much more efficient than using (10) which requires $\left(n^{3}+6 n^{2}-n\right) / 3$ operations.

Example 3 The matrix

$$
\boldsymbol{A}=\left[\begin{array}{rrrrr}
3.4082 & -0.0794 & -0.4425 & -0.2089 & -0.9685 \\
-0.0794 & 2.9171 & -0.6922 & -0.3937 & -0.3219 \\
-0.4425 & -0.6922 & 2.8730 & 0.8300 & -1.1523 \\
-0.2089 & -0.3937 & 0.8300 & 2.7639 & -0.9687 \\
-0.9685 & -0.3219 & -1.1523 & -0.9687 & 3.0378
\end{array}\right]
$$

has eigenvalues ${ }^{1}\{1.0000,2.0000,3.0000,4.0000,5.0000\}$. We determine a vector $\boldsymbol{v}$ such that the matrix $\boldsymbol{A}+\boldsymbol{v} \boldsymbol{v}^{T}$ has eigenvalues

$$
\{1.1093,0.7395,0.5929,0.4841,0.3698\} \text {. }
$$

${ }^{1}$ All calculations for this article were performed in Matlab using IEEE standard double precision arithmetic with machine epsilon $\approx 2.22 \times 10^{-16}$. Results are correctly rounded to the number of figures shown. 
Using (12) we get (taking all the square roots to be positive),

$$
\hat{\boldsymbol{v}}=(1.2305,0.5469,0.3516,0.2344,0.1367)^{T}
$$

from which we get $\boldsymbol{v}=\boldsymbol{Q} \hat{\boldsymbol{v}}$ to be $(-0.3060,0.8401,0.9474,-0.3548,0.8230)^{T}$. The eigenvalues of the matrix $\boldsymbol{A}+\sigma \boldsymbol{v} \boldsymbol{v}^{T}$ are, correctly rounded to the number of figures shown, the set (13).

Let us now consider partial pole assignment for a single matrix.

Let $S=\left\{i_{1}, i_{2}, \ldots, i_{r}\right\}$ be an ordered $\left(i_{j} \leq i_{k}\right.$ if $\left.j \leq k\right)$ subset of the integers $1,2, \ldots, n, r \leq n$ and let $T$ be the complement of $S$.

Suppose $\left\{\mu_{i}\right\}_{i \in S}$ is a set of $r$ scalars which satisfy

$$
\begin{aligned}
& \lambda_{i} \leq \mu_{i} \leq \lambda_{i+1}, i \in S, \quad \text { if } \sigma=1 \text { and } \\
& \mu_{i} \leq \lambda_{i} \leq \mu_{i+1}, i \in S, \quad \text { if } \sigma=-1 .
\end{aligned}
$$

To assign only $\left\{\mu_{i}\right\}_{i \in S}$ while leaving $\left\{\lambda_{i}\right\}_{i \in T}$ unchanged we simply set to zero the elements of the vector $\hat{\boldsymbol{v}}$ which correspond to those poles we want to leave unchanged, $\hat{v}_{i}=0$ if $i \in T$. For each component of $\hat{\boldsymbol{v}}$ which is zero the secular equation has one fewer term and the corresponding Cauchy matrix has one fewer row and column. Thus, (12) is now replaced by

$$
\hat{v}_{i}^{2}=-\frac{\prod_{k \in S}\left(\lambda_{i}-\mu_{k}\right)}{\prod_{k \in S, k \neq i}\left(\lambda_{i}-\lambda_{k}\right)}, \quad i \in S .
$$

In all other respects the process is unchanged.

Example 4 Using the matrix $\boldsymbol{A}$ of Example 3 we construct a vector $\boldsymbol{v}$ such that the matrix $\boldsymbol{A}+\boldsymbol{v} \boldsymbol{v}^{T}$ has eigenvalues

$$
\{1.5000,2.0000,3.5000,4.0000,5.5000\} \text {. }
$$


In other words we assign the first, third and fifth eigenvalues and leave the second and fourth eigenvalues unchanged.

The indices in formula (15) now run over only the values $1,3,5$. In other words, the formula only includes the eigenvalues $\lambda_{j}$ of $\boldsymbol{A}$ which are to be reassigned and the eigenvalues $\mu_{j}$ which replace them. The vector $\hat{\boldsymbol{v}}$ which we get is (again taking all square roots to be positive) now $\hat{\boldsymbol{v}}=(0.8385,0.0000,0.6847,0.0000,0.5728)^{T}$ from which we recover $\boldsymbol{v}=\boldsymbol{Q} \hat{\boldsymbol{v}}$ as $\boldsymbol{v}=(-0.0621,0.9936,0.1184,-0.0089,0.7034)^{T}$. From the choices that are available for the signs of the square roots of the $\hat{v}_{i}^{2}$ we see that there are eight different solution possible here. The eigenvalues of the matrix $\boldsymbol{A}+\boldsymbol{v} \boldsymbol{v}^{T}$ are, to the number of figures shown, the set (16).

\section{Symmetry preserving pole assignment for a symmetric pair of matrices}

In this section we consider the symmetric definite pencil $P(\lambda)=\boldsymbol{A}-\lambda \boldsymbol{B}$, $\boldsymbol{A}, \boldsymbol{B} \in \mathbb{R}^{n \times n}$ and show how to assign all or a part of its spectrum by a symmetry preserving rank-one update while leaving unchanged those eigenvalues not replaced. We first briefly review the derivation of a secular equation for this generalized eigenvalue problem.

The real scalar $\lambda$ and the associated vector $\boldsymbol{y}$ which satisfy $(\boldsymbol{A}-\lambda \boldsymbol{B}) \boldsymbol{y}=$ $\mathbf{0}, \quad \boldsymbol{y}^{T} \boldsymbol{B} \boldsymbol{y}=1$ are called an eigenvalue and normalized eigenvector of $P(\lambda)$. Let $\boldsymbol{Y}$, regular, be the matrix which simultaneously diagonalizes $\boldsymbol{A}$ and $\boldsymbol{B}$ as

$$
\boldsymbol{Y}^{T} \boldsymbol{A} \boldsymbol{Y}=\boldsymbol{\Lambda}=\operatorname{diag}\left\{\lambda_{1}, \lambda_{2}, \ldots, \lambda_{n}\right\}, \quad \boldsymbol{Y}^{T} \boldsymbol{B} \boldsymbol{Y}=\boldsymbol{I} .
$$

For a given vector $\boldsymbol{u} \in \mathbb{R}^{n}$ and the two real scalars $\alpha$ and $\beta$, we define the eigenvector $\boldsymbol{x}$ and its corresponding eigenvalue $\mu$ as those which satisfy

$$
\left(\boldsymbol{A}+\alpha \boldsymbol{u} \boldsymbol{u}^{T}\right) \boldsymbol{x}=\mu\left(\boldsymbol{B}+\beta \boldsymbol{u} \boldsymbol{u}^{T}\right) \boldsymbol{x}, \quad \boldsymbol{x}^{T}\left(\boldsymbol{B}+\beta \boldsymbol{u} \boldsymbol{u}^{T}\right) \boldsymbol{x}=1 .
$$


Multiplying (18) on the left by $\boldsymbol{Y}^{T}$ gives $\boldsymbol{Y}^{T}\left(\boldsymbol{A}+\alpha \boldsymbol{u} \boldsymbol{u}^{T}\right) \boldsymbol{Y} \boldsymbol{Y}^{-1} \boldsymbol{x}=$ $\mu \boldsymbol{Y}^{T}\left(\boldsymbol{B}+\beta \boldsymbol{u} \boldsymbol{u}^{T}\right) \boldsymbol{Y} \boldsymbol{Y}^{-1} \boldsymbol{x}$, from which $\left(\boldsymbol{\Lambda}+\alpha \boldsymbol{Y}^{T} \boldsymbol{u} \boldsymbol{u}^{T} \boldsymbol{Y}\right)\left(\boldsymbol{Y}^{-1} \boldsymbol{x}\right)=\mu(\boldsymbol{I}+$ $\left.\beta \boldsymbol{Y}^{T} \boldsymbol{u} \boldsymbol{u}^{T} \boldsymbol{Y}\right)\left(\boldsymbol{Y}^{-1} \boldsymbol{x}\right)$ follows. Setting $\hat{\boldsymbol{u}}=\boldsymbol{Y}^{T} \boldsymbol{u}$ and $\boldsymbol{Y} \hat{\boldsymbol{x}}=\boldsymbol{x}$ gives [2] $\left(\boldsymbol{\Lambda}+\alpha \hat{\boldsymbol{u}} \hat{\boldsymbol{u}}^{T}\right) \hat{\boldsymbol{x}}=\mu\left(\boldsymbol{I}+\beta \hat{\boldsymbol{u}} \hat{\boldsymbol{u}}^{T}\right) \hat{\boldsymbol{x}}$.

Lemma 5 Suppose the $\lambda_{j}$ of (5) are distinct and $\boldsymbol{Y}$ is such that $\lambda_{1}<\lambda_{2}<$ $\cdots<\lambda_{n}$. Assume further, that $\boldsymbol{e}_{j}^{T} \hat{\boldsymbol{u}} \neq 0$ and $\lambda_{j} \neq \alpha / \beta$ for all $j$. Then, (a) $(\mu \boldsymbol{I}-\boldsymbol{\Lambda})$ is invertible, (b) $\mu \neq \alpha / \beta$, (c) $\hat{\boldsymbol{x}}^{T} \hat{\boldsymbol{u}} \neq 0$.

Furthermore, the eigenvalues of the pair $(\boldsymbol{A}, \boldsymbol{B})$, even in the absence of the distinctness assumption, and those of the modified pair $\left(\boldsymbol{A}+\alpha \boldsymbol{u} \boldsymbol{u}^{T}, \boldsymbol{B}+\right.$ $\left.\beta \boldsymbol{u} \boldsymbol{u}^{T}\right)$ are related by a secular equation that is the counterpart of relation (8) for this case $(\alpha-\beta \mu) \hat{\boldsymbol{u}}^{T}(\mu \boldsymbol{I}-\boldsymbol{\Lambda})^{-1} \hat{\boldsymbol{u}}=1$ sometimes written as

$$
(\alpha-\beta \mu) \sum_{j=1}^{n} \frac{\hat{u}_{j}^{2}}{\mu-\lambda_{j}}=1 .
$$

Note, as for the standard eigenvalue problem, that if $\boldsymbol{e}_{j}^{T} \hat{\boldsymbol{u}}=0$ for some $j$ then $\left(\lambda_{j}, \boldsymbol{e}_{j}\right)$ is an eigenpair of the modified system because then $(\boldsymbol{\Lambda}+$ $\left.\alpha \hat{\boldsymbol{u}} \hat{\boldsymbol{u}}^{T}\right) \boldsymbol{e}_{j}-\mu\left(\boldsymbol{I}+\beta \hat{\boldsymbol{u}} \hat{\boldsymbol{u}}^{T}\right) \boldsymbol{e}_{j}=\boldsymbol{\Lambda} \boldsymbol{e}_{j}-\lambda_{j} \boldsymbol{e}_{j}=\mathbf{0}$. Once again, we can deflate the problem in this case to one with dimension $n-1$ and the secular equation then has $n-1$ terms and $n-1$ solutions and so the modification has left the eigenvalue $\lambda_{j}$ of the original system unchanged.

We apply the same strategy here in the generalized eigenvalue problem that we used in the standard eigenvalue case for full and partial pole assignment. We use relation (19) to construct a system of linear equations which can be used to determine the components $\hat{u}_{j}$ from which we find the symmetric rank-one modification that should be applied to matrices $\boldsymbol{A}$ and $\boldsymbol{B}$ to assign part or all of their spectrum.

For the full pole assignment problem the matrix system is as in (10) 
except that we now have the right-hand side

$$
\left(\frac{1}{\alpha-\beta \mu_{1}}, \frac{1}{\alpha-\beta \mu_{2}}, \ldots, \frac{1}{\alpha-\beta \mu_{n}}\right)^{T} .
$$

Using the identity (11) the explicit solution for this case is

$$
\hat{u}_{i}^{2}=\prod_{k=1}^{n} \frac{\left(\lambda_{i}-\mu_{k}\right)}{\left(\beta \mu_{k}-\alpha\right)} \prod_{k=1, k \neq i}^{n} \frac{\left(\beta \lambda_{k}-\alpha\right)}{\left(\lambda_{i}-\lambda_{k}\right)}, \quad i=1,2, \ldots, n .
$$

Here again, there are $2^{n}$ different solutions in view of the two sign choices that can be made for each component of the solution vector $\hat{\boldsymbol{u}}$.

If we consider only $\times, \div$ operations then using $(20)$ costs $6 n(n-1)$ operations and so is computationally much more efficient than solving the matrix system (10) with the altered right-hand-side since this requires $\left(n^{3}+6 n^{2}+\right.$ $2 n) / 3$ operations.

As before we solve the partial pole placement problem by dealing with a reduced system of equations which defines the non-zero elements of the required vector $\hat{\boldsymbol{u}}$ : we simply set to zero the elements of the vector $\hat{\boldsymbol{u}}$ which correspond to those poles we want to leave unchanged. For each component of $\hat{\boldsymbol{u}}$ which is zero the secular equation has one fewer term and the corresponding Cauchy matrix has one fewer row and column. In the explicit solution formula for this case the variable $k$ in (20) ranges only over the indices of the eigenvalues to be changed.

More precisely, let the sets of indices $S$ and $T$ be defined as before and let $\alpha, \beta,\left\{\lambda_{j}\right\}_{1}^{n}$, and $\left\{\mu_{j}\right\}_{1}^{n}$ satisfy the interlacing property (6). We set $\hat{u}_{i}=0$ for all $i \in T$ and compute

$$
\hat{u}_{i}^{2}=\prod_{k \in S} \frac{\left(\lambda_{i}-\mu_{k}\right)}{\left(\beta \mu_{k}-\alpha\right)} \prod_{k \in S, k \neq i}^{n} \frac{\left(\beta \lambda_{k}-\alpha\right)}{\left(\lambda_{i}-\lambda_{k}\right)},, \quad i \in S .
$$


3 Symmetry preserving pole assignment for a symmetric pair of matrices $\mathrm{C} 276$

The following example illustrates partial pole assignment using this method. The generalization to full assignment is clear.

Example 6 We consider the matrix pair

$$
\boldsymbol{A}=\left[\begin{array}{lllll}
0.1752 & 0.1121 & 0.1249 & 0.2070 & 0.1290 \\
0.1121 & 0.1322 & 0.0799 & 0.1298 & 0.1219 \\
0.1249 & 0.0799 & 0.0998 & 0.1690 & 0.1199 \\
0.2070 & 0.1298 & 0.1690 & 0.2922 & 0.2020 \\
0.1290 & 0.1219 & 0.1199 & 0.2020 & 0.2021
\end{array}\right]
$$

and

$$
\boldsymbol{B}=\left[\begin{array}{lllll}
0.2218 & 0.2110 & 0.1076 & 0.1311 & 0.1217 \\
0.2110 & 0.3015 & 0.1625 & 0.1413 & 0.1837 \\
0.1076 & 0.1625 & 0.1314 & 0.1250 & 0.1256 \\
0.1311 & 0.1413 & 0.1250 & 0.1505 & 0.1026 \\
0.1217 & 0.1837 & 0.1256 & 0.1026 & 0.1686
\end{array}\right]
$$

which has eigenvalues $\left\{\lambda_{j}\right\}_{j=1}^{5}$

$$
\{0.0045,0.1762,0.6012,2.4847,10.1337\} .
$$

We choose $\alpha=0.4939$ and $\beta=0.4175$ and so, in order to satisfy the constraints in (6), any eigenvalues $\mu_{j}$ which will replace the existing eigenvalues $\lambda_{j}$ must interlace the set comprising the numbers in (22) and the ratio $\alpha / \beta=1.1830$. We replace the first, third and fifth eigenvalues shown in (22) with the numbers

$$
\mu_{1}=0.0906, \quad \mu_{3}=0.8900, \quad \mu_{5}=6.2905 .
$$

As before the second and fourth components of $\hat{\boldsymbol{u}}$ are set to zero and the three other components are computed by (21) with the $S=\{1,3,5\}$ and $T=\{2,4\}$. Alternatively, we could compute the non-zero components of $\hat{\boldsymbol{u}}$ as the solution of the three by three system derived from the original matrix 
system by deleting the second and fourth rows and columns. In either case we get (taking all square roots to be positive)

$$
\hat{\boldsymbol{u}}=(0.7776,0.0000,1.5098,0.0000,1.9266)^{T},
$$

and then recover $\boldsymbol{u}=(0.4884,0.3361,0.0552,0.3385,0.0190)^{T}$ as the solution of $\boldsymbol{Y}^{T} \boldsymbol{u}=\hat{\boldsymbol{u}}$. The eigenvalues of the modified pair $\left(\boldsymbol{A}+\alpha \boldsymbol{u} \boldsymbol{u}^{T}, \boldsymbol{B}+\beta \boldsymbol{u} \boldsymbol{u}^{T}\right)$ are, correctly rounded to the number of figures shown, the three numbers in (23) and the second and fourth numbers of (22).

\section{Conclusions}

We presented a new explicit solution to the symmetry preserving partial and full pole assignment problem for the symmetric definite matrix pair and have shown how symmetry preserving partial pole assignment can be done for the standard eigenvalue problem. Numerical testing of the methods, to be presented in a separate report, suggests that, even for matrices of dimension 1024 with the prescribed poles extremely close to the existing poles, IEEE double precision arithmetic can deliver about ten decimals of relative accuracy.

\section{References}

[1] B. N. Datta. Finite element model updating and partial eigenvalue assignment in structural dynamics: recent developments on computational methods. volume Proceedings of the 10th International Conference MMA2005 and CMAM2, Trakai, pages 15-27, 2005. http://www.math.niu.edu/ dattab/psfiles/updating.ps C266

[2] S. Elhay, G. H. Golub, and Y. M. Ram. On the spectrum of a modified linear pencil. Computers and Maths with Appl., 46:1413-1426, 2003. 
http://www.cs.adelaide.edu.au/users/elhay/psfiles/rmsm.ps $\mathrm{C} 267, \mathrm{C} 274$

[3] G. H. Golub and C. F. Van Loan. Matrix computations. North Oxford Academic Publishing, 2nd edition, 1989. C266, C269

[4] M. Gu and S. Eisenstat. A stable and efficient algorithm for the rank-one modification of the symmetric eigenproblem. SIAM J. Matrix Anal. Appl., 15(4):1266-1276, 1994.

http: //portal .acm.org/citation. $c f m ? i d=196045.196076 \& d l=\& d l=$ \&CFID $=15151515 \&$ CFTOKEN=6184618 C270

[5] D. Knuth. The Art of Computer Programming, volume 1/Fundamental Algorithms. Addison-Wesley, Reading, Massachusetts, 1981. 2nd Edition. C271

[6] K. Lowner. Uber monotone matrixfunktionen. Math. Z., 38:177-216, 1934. C268

[7] Y. M. Ram. Pole assignment for the vibrating rod. Q. J. Mech. and Appl. Math., 51(3):477-492, 1998. http:

//qjmam.oxfordjournals.org/cgi/content/abstract/51/3/461 $\mathrm{C} 266$ 


\section{Author address}

1. S. Elhay, School of Computer Science, University of Adelaide, South Australia 5005.

mailto:sylvan.elhay@adelaide.edu.au 